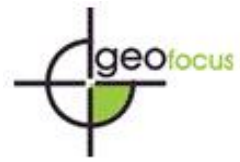

Campos Nazer, J., Espinoza Aburto, J., Ramos Aguilar, J. (2021). Estudio espaciotemporal de la vegetación existente y su relación con el desierto florido en la región de Atacama, Chile. GeoFocus, Revista Internacional de Ciencia y Tecnología de la Información Geográfica (Artículos), 28, 83-93. http://dx.doi.org/10.21138/GF.722

\title{
ESTUDIO ESPACIOTEMPORAL DE LA VEGETACIÓN EXISTENTE Y SU RELACIÓN CON EL DESIERTO FLORIDO EN LA REGIÓN DE ATACAMA, CHILE
}

\author{
${ }^{1}$ JUAN CAMPOS NAZER, 2JOSÉ ESPINOZA ABURTO, 3JORGE RAMOS AGUILAR, \\ ${ }^{1}$ Área de Geominería, Universidad de Atacama. \\ Copayapu 485 - Copiapó - Chile \\ ${ }^{2}$ Junta de Vigilancia de la Cuenca del Rio Huasco y Sus Afluentes. \\ Arturo Prat 202, Vallenar, Atacama, Chile \\ ${ }^{3}$ Pontificia Universidad Católica de Chile \\ ${ }^{3}$ Av Libertador Bernardo O'Higgins 340, Santiago, Región Metropolitana, Chile \\ 1juan.campos@uda.cl, ${ }^{2}$ juan.campos@uda.cl, ${ }^{3}$ jsramos@uc.cl
}

\section{RESUMEN}

En la Región de Atacama, Chile, se desarrolla el fenómeno "desierto florido", el cual es el resultado de eventos de precipitación infrecuentes, que regulan la aparición de la biota existente. Se determinó la distribución espacial y temporal de la vegetación en esta región desde el año 2000 hasta el 2017. La cobertura vegetal se determinó a través del producto MOD13Q1 que deriva del sensor MODIS. Este producto utiliza el índice NDVI. El procesamiento de la información se realizó mediante Python y la librería ArcPy. Posteriormente se generaron gráficas del factor NDVI, además de mapas de cobertura de vegetación anuales. Los resultados arrojaron que el 2017 fue un año con una considerable cubierta vegetal, con un $8 \%$ más de vegetación respecto al 2015 , los cuales son años de registro de gran cobertura vegetal según Corporación Nacional Forestal de Chile (CONAF). El factor NDVI máximo se concentra el año 2017, el mayor en los últimos 18 años. Esta cantidad de vegetación se ve incrementada entre mayo a julio principalmente por la presencia de floración relacionada al desierto florido, evento que se desarrolló debido a precipitaciones abundantes ocurridas durante mayo del mismo año.

Palabras clave: desierto florido; NDVI; MODIS; SIG; Terra.

\section{SPATIOTEMPORAL STUDY OF EXITING VEGETATION IN THE ATACAMA REGION} AND ITS RELATIONSHIP WITH FLOWERING DESERT.

\section{ABSTRACT}

In the Atacama Region, Chile, a phenomenon known as the "flowering desert" (or "desert bloom") occurs, which is the result of infrequent precipitation events that regulate the amount of existing biota. In this context, this study graphs the spatial and temporal distribution 
Campos Nazer, J., Espinoza Aburto, J., Ramos Aguilar, J. (2021). Estudio espaciotemporal de la vegetación existente y su relación con el desierto florido en la región de Atacama, Chile. GeoFocus, Revista Internacional de Ciencia y Tecnología de la Información Geográfica (Artículos), 28, 59-93. http://dx.doi.org/10.21138/GF.722

of vegetation in the Region from 2000 to 2017. Vegetation areas have been determined using the MOD13Qproduct derived from the MODIS sensor. This product uses the NDVI factor, which detects vegetation vigor. For the analysis, the ArcPy library written in Python was used. Later graphs of the NDVI factor were generate, in addition to annual vegetation density maps of the vegetal cover. The results showed that 2017 was a year with $8 \%$ more vegetation compared to 2015, being those years among the ones with larger vegetation cover according to Chilean Corporación Nacional Forestal (CONAF). The maximum NDVI factor was concentrated in 2017, making this year the one with the highest amount of concentrated vegetation in the last 18 years. This amount of vegetation is increased between May to July mainly due to the presence of flowering related to the Flowering Desert which triples the surface of vegetation. This higher concentration of vegetation would not have been possible without the abundant rainfall that occurred during May of 2017.

Keywords: flowering desert; NDVI; MODIS; GIS; Terra.

\section{Introducción}

La región de Atacama es parte del límite meridional del desierto de Atacama. El ecosistema dominante es desértico, donde la productividad es baja, altamente variable y dependiente de las precipitaciones y la disponibilidad de nutrientes y porque la riqueza de especies es baja y está correlacionada con las precipitaciones, entre otras características (NoyMeir, 1973). Dentro de la región podemos encontrar 4 tipos de climas que moldean este ecosistema: 1) Desértico costero con nubosidad abundante correspondiente a la franja costera entre Chañaral (26 $21^{\prime}$ S, $70^{\circ} 37^{\prime}$ O) y Quebrada de los Choros $\left(29^{\circ} 20^{\prime}\right.$ S S), 2) Desértico transicional, entre el límite de la franja costera hasta los $1500 \mathrm{msnm}$, 3) Desértico frío de montaña, entre los $1500 \mathrm{msnm}$ y los $4000 \mathrm{msnm}$ y 4) Tundra de alta montaña, hasta los 4500 msnm (Juliá et al., 2008). Como es de esperar, cada uno de estos presentan diferencias en las precipitaciones. Estas se incrementan a mayor latitud y altitud, como lo evidencian los registros de Chañaral $\left(26^{\circ} 20^{\prime} \mathrm{S}, 70^{\circ} 36^{\prime} \mathrm{O}\right)$ con $1,7 \mathrm{~mm} / \mathrm{año}$, Potrerillos $\left(26^{\circ} 22^{\prime} \mathrm{S}, 6^{\circ} 28^{\prime} \mathrm{O}\right)$ con $52 \mathrm{~mm} /$ año, Freirina $\left(28^{\circ} 30^{\prime} \mathrm{S}, 7^{\circ} 04^{\prime} \mathrm{O}\right)$ con $42 \mathrm{~mm} /$ año y El Tránsito $\left(28^{\circ} 53^{\prime} \mathrm{S}, 70^{\circ} 17^{\prime}\right.$ O) con $85 \mathrm{~mm} / \mathrm{año} \mathrm{(Almeyda} \mathrm{\&} \mathrm{Sáez,} \mathrm{1958;} \mathrm{Di} \mathrm{Castri} \mathrm{\&} \mathrm{Hajek,} \mathrm{1976),} \mathrm{consideradas}$ precipitación media anual.

Estas precipitaciones, escasas e infrecuentes, elevan la humedad del suelo que regula la cantidad y disponibilidad de la biota existente en el desierto (Noy-Meir, 1973). Después de la lluvia, extensión de vegetación es extremadamente significativa (Juliá et al., 2008), lo que sustenta un conjunto biótico rico, pero de vida corta. Una importante proporción de esta biota permanece inactiva durante los períodos intermedios entre lluvias (Vidiella et al., 1999). Las especies de plantas que responden a estos eventos de lluvias son principalmente anuales de vida corta, geófitas bulbosas y arbustos con dormancia de verano (Armesto et al., 1993). El inusual y explosivo desarrollo de estas especies herbáceas después de la lluvia es conocido como el desierto florido (Armesto et al., 1993; Jaksic, 2001). Este incremento repentino en la producción primaria neta trae consigo cambios relevantes en el ecosistema, tales como aparición de roedores y depredadores, polinizadores, etc. (Jaksic, 2001). Se ha demostrado empíricamente que, para algunas especies, las semillas en dormancia necesitan un mínimo de $15 \mathrm{~mm}$ de precipitación en un año para iniciar el proceso de germinación (Armesto et al., 1993; Vidiella et al., 1999). Debido a los tipos de clima presentes, esta cantidad es común en la región. Sin 


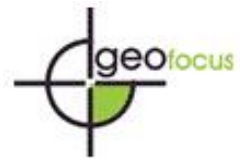

Campos Nazer, J., Espinoza Aburto, J., Ramos Aguilar, J. (2021). Estudio espaciotemporal de la vegetación existente y su relación con el desierto florido en la región de Atacama, Chile. GeoFocus, Revista Internacional de Ciencia y Tecnología de la Información Geográfica (Artículos), 28, 59-93. http://dx.doi.org/10.21138/GF.722

embargo, estas precipitaciones ocurren en eventos climáticos extremos o en años con presencia de El Niño - Oscilación del Sur (Jaksic, 2001). De hecho, el fenómeno del desierto florido ha sido reportado en años con eventos de El Niño - Oscilación del Sur más intensos de lo normal (Rayner et al., 2003), trabajos Ortlieb han comparado eventos de El Niño con el desierto florido en los últimos dos siglos, encontrando una correlación de estos dos últimos.

En el periodo invernal de 2017 se produjo un evento meteorológico que dejó en solo 3 días $20 \mathrm{~mm}$ en Copiapó y más de $40 \mathrm{~mm}$ en Vallenar (Dirección de Meteorología de Chile, 2018), las cuales afectaron directamente a las áreas en donde se produce el desierto florido (Chávez et al., 2019), aportando de manera significativa al desarrollo de la cobertura vegetal de la zona. Este estudio se realizó en base a ese evento.

A pesar de que el fenómeno del desierto florido es considerado relevante para botánicos, ecólogos y geólogos (Jaksic, 2001) hay pocos estudios sobre los patrones espaciotemporales o que mapeen la extensión de este. Entre ellos destacan Castro et al. (2014), quienes determinaron la distribución espacial del desierto florido en el Mar de Dunas en el Noroeste de Copiapó; He et al. (2017) quienes cuantificaron el efecto de enfriamiento de un área con vegetación en el desierto de Atacama y Chávez et al. (2019) quienes identificaron los eventos históricos de desierto florido entre 1981 y 2015 y sus patrones espaciotemporales.

El objetivo general de este estudio es revisar variaciones del índice NDVI en relación a zonas normalmente desprovistas de vegetación, asociando estos cambios al fenómeno desierto florido, por lo que se revisarán puntos in situ para verificar esta hipótesis, considerando imágenes desde el año 2000 al 2017 en la región de Atacama

\section{Materiales, datos y métodos}

Debido a que el estudio pretende identificar como el desierto florido se emplaza en la superficie de la región de Atacama, ubicada entre los $26^{\circ}$ y $29^{\circ} 20^{\prime}$ latitud sur, Chile, y en qué intervalos de tiempo se presenta, este tipo de estudio requiere de datos ráster que tengan información de la vegetación, además de un alcance espacial a nivel regional y de una resolución temporal de máximo 15 días. Estas características las tiene el producto MOD13Q1 V006 del sensor MODIS (Moderate Resolution Imaging Spectroradiometer), ubicado en el satélite Terra de la NASA. Este producto ha sido diseñado por en el marco del programa EOS de la NASA, para dar continuidad al NDVI del programa NOAA AVHRR, el cual es generado a escala mundial con una resolución espacial de 250 metros de píxel y una resolución temporal de 16 días, un sistema de coordenadas de tipo sinusoidal MODIS TILE SIN y un área que cubre 1200 kilómetros por granulo. Debido a que el satélite fue puesto en marcha en febrero del año 2000, la disponibilidad de este producto abarca desde el 18 de febrero de ese año al 2017.

\subsection{Datos Satelitales}

Para este estudio se realizaron descargas de imágenes satelitales del sensor MODIS, el cual se encuentra a bordo de los satélites espaciales Terra lanzados por la NASA, los cuales entregan una imagen satelital diaria de la superficie terrestre. Para esto se utilizó la herramienta GNU WGET la cual, mediante el lenguaje de programación Python, permite la descarga masiva de archivos desde los servidores de la NASA.

\subsection{Satélite Terra}




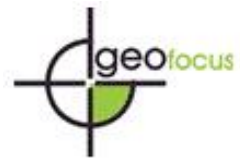

Campos Nazer, J., Espinoza Aburto, J., Ramos Aguilar, J. (2021). Estudio espaciotemporal de la vegetación existente y su relación con el desierto florido en la región de Atacama, Chile. GeoFocus, Revista Internacional de Ciencia y Tecnología de la Información Geográfica (Artículos), 28, 59-93. http://dx.doi.org/10.21138/GF.722

El Satélite Terra forma parte de una misión que incluye a EE.-UU., Canadá y Japón. Una de sus grandes características es la capacidad que tiene para registrar los cambios climáticos de la atmósfera con la precisión adecuada como para permitir un pronóstico a corto plazo. Terra gira alrededor de la Tierra a $705 \mathrm{~km}$ de altura, de manera sincronizada con el sol y va desde polo norte a polo sur. Su vida útil se estima en 6 años y dado que se concibió como un centro de investigaciones, lleva a bordo cinco instrumentos: ASTER, CERES, MODIS, MISR, MOPITT.

\subsection{Sensor MODIS}

MODIS corresponde a un sensor para la teledetección que contiene distintas características espaciales y espectrales y es uno de los más importantes para estudiar los cambios en la superficie de la tierra. Este fue diseñado por un equipo interdisciplinario de científicos con una vasta experiencia en sensores remotos (Mas, 2011). Este equipo trabajó por cerca de 10 años para finalmente definir los requisitos para la toma, calibración y procesamiento de los datos (Lyapustin et al., 2014).

El sensor MODIS se encuentra a bordo de los satélites TERRA o EOS-AM el cual fue lanzado en diciembre del año 1999 y AQUA o EOS-PM el cual fue lanzado en mayo del 2002. La órbita de ambas plataformas es helio-sincrónica y cuasi-polar con una inclinación de $98.2^{\circ}$ y $98^{\circ}$ y una altitud media de 708 y $705 \mathrm{~km}$ respectivamente (NASA-MODIS, 2010), con el fin de adquirir datos para el conocimiento de la dinámica atmosférica global y la interacción tierraocéano-atmósfera (Engel-Cox et al., 2004). Terra está programada para pasar de norte a sur cruzando el ecuador a las 10:30 hrs. de la mañana en su órbita descendente, mientras que Aqua pasa de sur a norte sobre el ecuador a las 1:30 hrs. de la tarde.

El formato del producto a trabajar es HDF-EOS "Hierarchical Data Format", el cual es de tipo matriz de dos dimensiones geográficas y 12 tipos de informaciones distintas, lo que hace necesaria su conversión a varios tipos de formatos para la creación de mapas y gráficos. El primer paso es extraer este formato matricial y traspasarlo a un formato que permita el procesamiento con software GIS o SIG (Sistema de Información Geográfica). Para el caso de este estudio se utilizaron las dos dimensiones, latitud con el valor "4800 (YDim: MODIS_Grid_16DAY_250m_500m_VI)", y longitud con el valor 4800 (XDim: MODIS_Grid_16DAY_250m_500m_VI), siendo finalmente el eje $\mathrm{Z}$ el valor llamado " $250 \mathrm{~m} 16$ days NDVI" que corresponde al valor NDVI con un tipo de dato "16-bit signedinteger" con valores desde el -2 000 a 10000 siendo el valor -3000 para el valor de relleno y un factor de escala de 0.0001 .

La extracción se realizó desde el formato HDF-EOS hacia un formato ráster, en este caso GEOTIF. Debido al gran número de archivos HDF-EOS, es necesario un algoritmo que realice la extracción de datos hacia el formato GEOTIF, para lo cual se utilizó la librería ArcPy en procesamiento por lotes, donde finalmente se obtuvieron los datos en formato GEOTIF.

El trabajo se realizó con una serie de pasos en software GIS, hasta la obtención de datos por píxel (véase figura 1). 


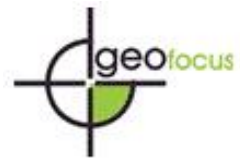

Campos Nazer, J., Espinoza Aburto, J., Ramos Aguilar, J. (2021). Estudio espaciotemporal de la vegetación existente y su relación con el desierto florido en la región de Atacama, Chile. GeoFocus, Revista Internacional de Ciencia y Tecnología de la Información Geográfica (Artículos), 28, 59-93. http://dx.doi.org/10.21138/GF.722

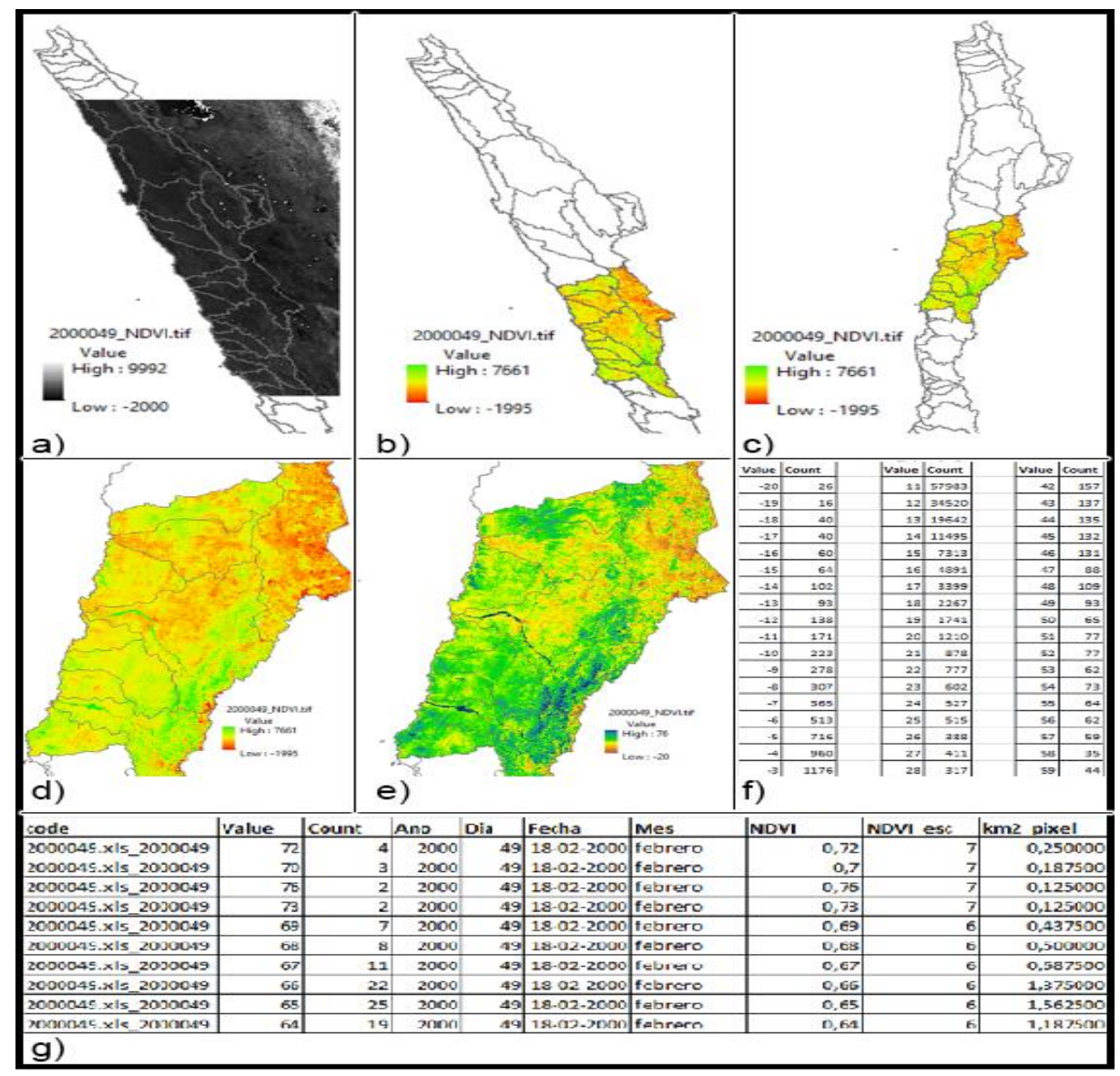

Figura 1. Pasos para la obtención de datos en software GIS

a) Información en formato HDF-EOS, b) datos en formato ráster, c) cambio en sistema de coordenadas, d) datos en formato escalado, e) Representación en paleta de colores estándar, f) extracción en formato de planilla de cálculo, g) integración final de datos en formato de planilla de cálculo.

*Value corresponde al índice NDVI sin decimales.

* $\mathrm{km}^{2}$ píxel, área donde existe índice NDVI en ese rango de valores.

Fuente: Elaboración propia

Tal como lo muestra la figura $1^{\text {a }}$, el área de estudio está situada en la Región de Atacama y la información ráster contiene un área mucho mayor. Esta área restante se eliminó realizando un corte en todas las imágenes ráster. Como se observa en la figura 1b, debido a la deformación presente en los datos, se hizo necesario un cambio en las coordenadas Sinusoidales MODIS TILE SIN hacia las coordenadas WGS84 Zona 19 Sur. Posteriormente se corrigió la escala de los datos NDVI haciendo uso de algebra de mapas hacia valores de NDVI 100 como máximo vigor de la vegetación y NDVI 20 como el mínimo, lo que corresponde a un ajuste para no procesar tantos datos en formato decimal. Valores inferiores representan suelos descubiertos sin vegetación. Esto es visible en la figura 1d y 1e. En la figura 1f, para tener información en gráficos de tiempo, se realizó un cambio entre estos datos geográficos y se transformaron a un formato acorde a este tipo de información, como lo es una planilla de cálculo y así se obtuvieron dos columnas: "Value" que es el valor NDVI y "Count" que es simplemente la cantidad de pixeles de un valor definido de NDVI que se encuentran en determinada imagen. Finalmente, mediante Macros de MS Excel se unieron todos los archivos para formar una pequeña base de 


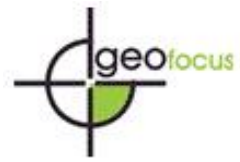

Campos Nazer, J., Espinoza Aburto, J., Ramos Aguilar, J. (2021). Estudio espaciotemporal de la vegetación existente y su relación con el desierto florido en la región de Atacama, Chile. GeoFocus, Revista Internacional de Ciencia y Tecnología de la Información Geográfica (Artículos), 28, 59-93. http://dx.doi.org/10.21138/GF.722

datos que se muestran en la figura $1 \mathrm{~g}$ con las siguientes columnas: "code" contiene la fecha de los datos obtenidos que para el procesamiento se extraen hacia las columnas Año, Día, Fecha, Mes; Value y Count contienen los datos anteriormente explicados. NDVI corresponde a Value*10-1. NDVI_esc corresponde a la decena * 10-1 del valor Value. $\mathrm{km}^{2}$ _pixel corresponde al área obtenida desde el sencillo cálculo $250 * 250 *$ Count, dato en kilómetros cuadrados. La visualización entrega el NDVI porcentajes (véase figura 2).

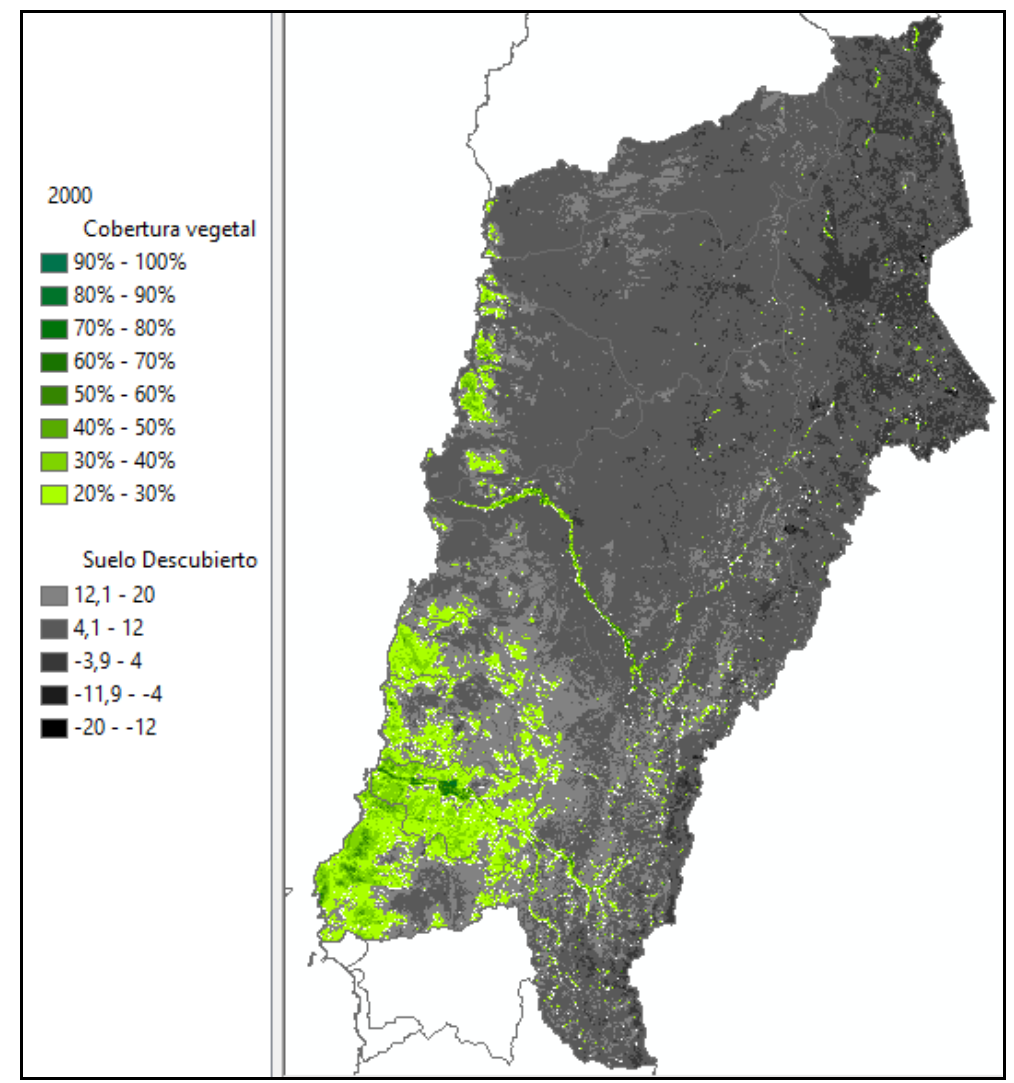

Figura 2. Modelo de cobertura vegetal de ejemplo para el año 2000.

Como se eliminaron los decimales, por efecto de procesamiento, lo valores quedaron concentrados en valores de 0 a 100, donde se filtraron píxeles con al menos $20 \%$ de cobertura, debido a que bajo este valor consideraba valores donde no existe vegetación.

Fuente: Elaboración propia.

Mediante algebra de mapas se calcularon los valores más altos de cada día y junto con un análisis en terreno se obtuvieron las áreas con mayor vegetación anual desde enero hasta agosto (véase figura 2). Luego se diseñaron los mapas para cada año con los valores en porcentaje de píxel de la cobertura vegetal anual. 


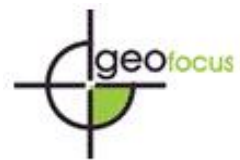

Campos Nazer, J., Espinoza Aburto, J., Ramos Aguilar, J. (2021). Estudio espaciotemporal de la vegetación existente y su relación con el desierto florido en la región de Atacama, Chile. GeoFocus, Revista Internacional de Ciencia y Tecnología de la Información Geográfica (Artículos), 28, 59-93. http://dx.doi.org/10.21138/GF.722

\section{Resultados y Discusión}

La cantidad de vegetación que se apreció según el índice NDVI, indica una gran extensión en el año 2017, muy por sobre los años anteriores (véase figura 3).

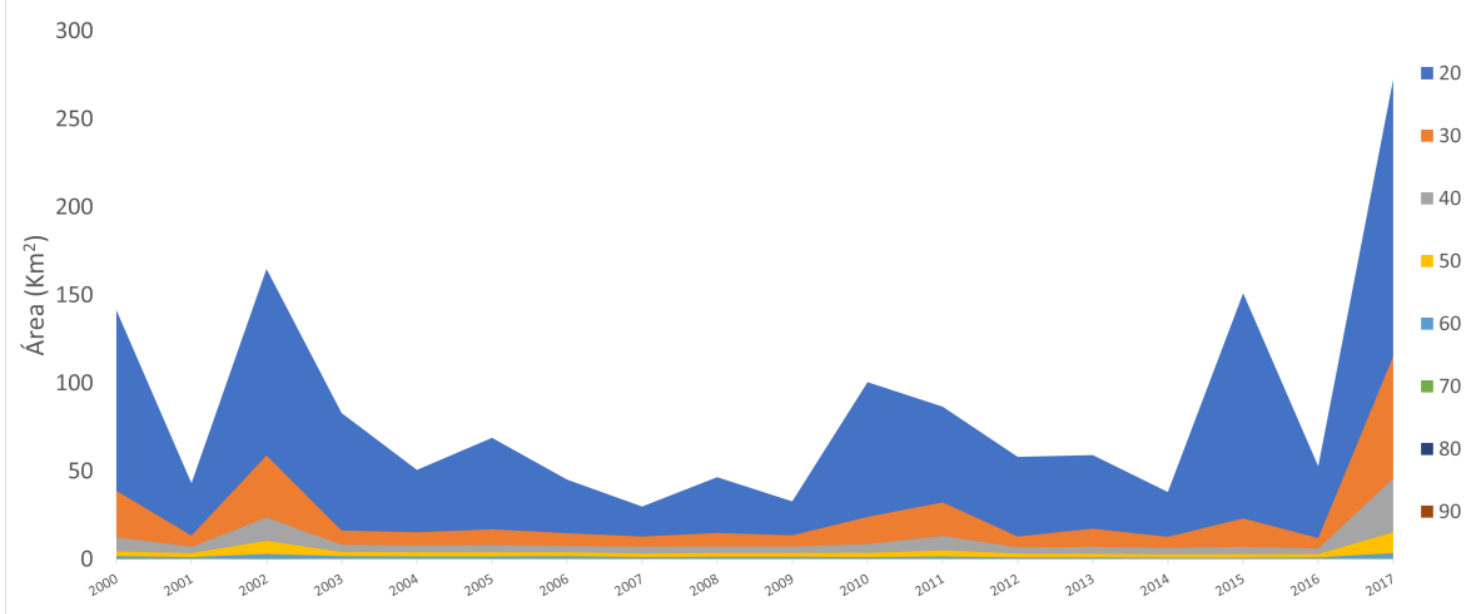

Figura 3. Vegetación total en la región de Atacama desde el año 2000 al año 2017. Fuente: Elaboración propia.

Además, los resultados indicaron que el año 2000, 2002 y 2015 fueron años que se presentó una importante abundancia de biota vegetal en la región de Atacama. No obstante, es inferior en comparación al año 2017.

La distribución de la vegetación, en los años 2000, 2002, 2011, 2015 y 2017, aumentó a partir del mes de junio, considerando información satelital, tal como se indica en la figura 4.

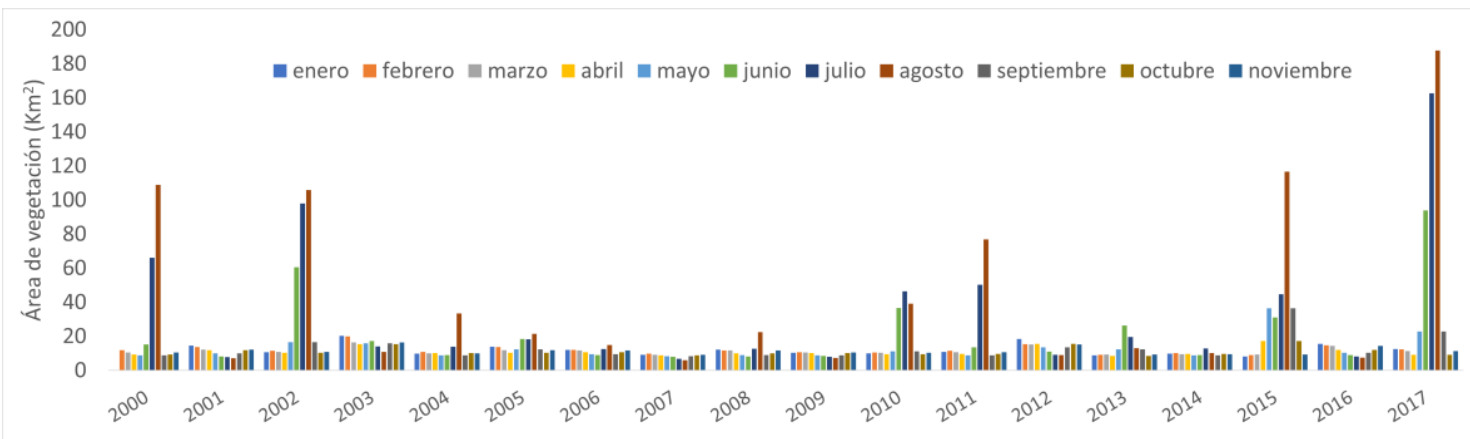

Figura 4. Distribución de la vegetación desde enero hasta agosto entre los años 2000 y 2017 Fuente: Elaboración propia.

Se obtuvieron datos de la estación de precipitaciones en Vallenar DGA ubicada en $28.5861^{\circ} \mathrm{S}$, $70.7397^{\circ} \mathrm{O}$, a una cota de $420 \mathrm{~m}$, Otra estación revisada es Canto del Agua DGA, $28.0992^{\circ} \mathrm{S}, 70.7811^{\circ} \mathrm{O}, 330 \mathrm{~m}$ y finalmente la estación Copiapó por su parte está ubicada en $27.0692^{\circ} \mathrm{S}, 70.8156^{\circ} \mathrm{O}, 15 \mathrm{~m}$, donde se obtuvo la variación de la suma de 


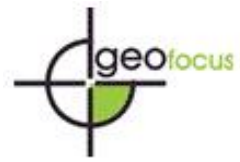

Campos Nazer, J., Espinoza Aburto, J., Ramos Aguilar, J. (2021). Estudio espaciotemporal de la vegetación existente y su relación con el desierto florido en la región de Atacama, Chile. GeoFocus, Revista Internacional de Ciencia y Tecnología de la Información Geográfica (Artículos), 28, 59-93. http://dx.doi.org/10.21138/GF.722

precipitaciones en mm entre el año 2014 y 2017, años donde también existió una fuerte fluctuación del desierto florido.

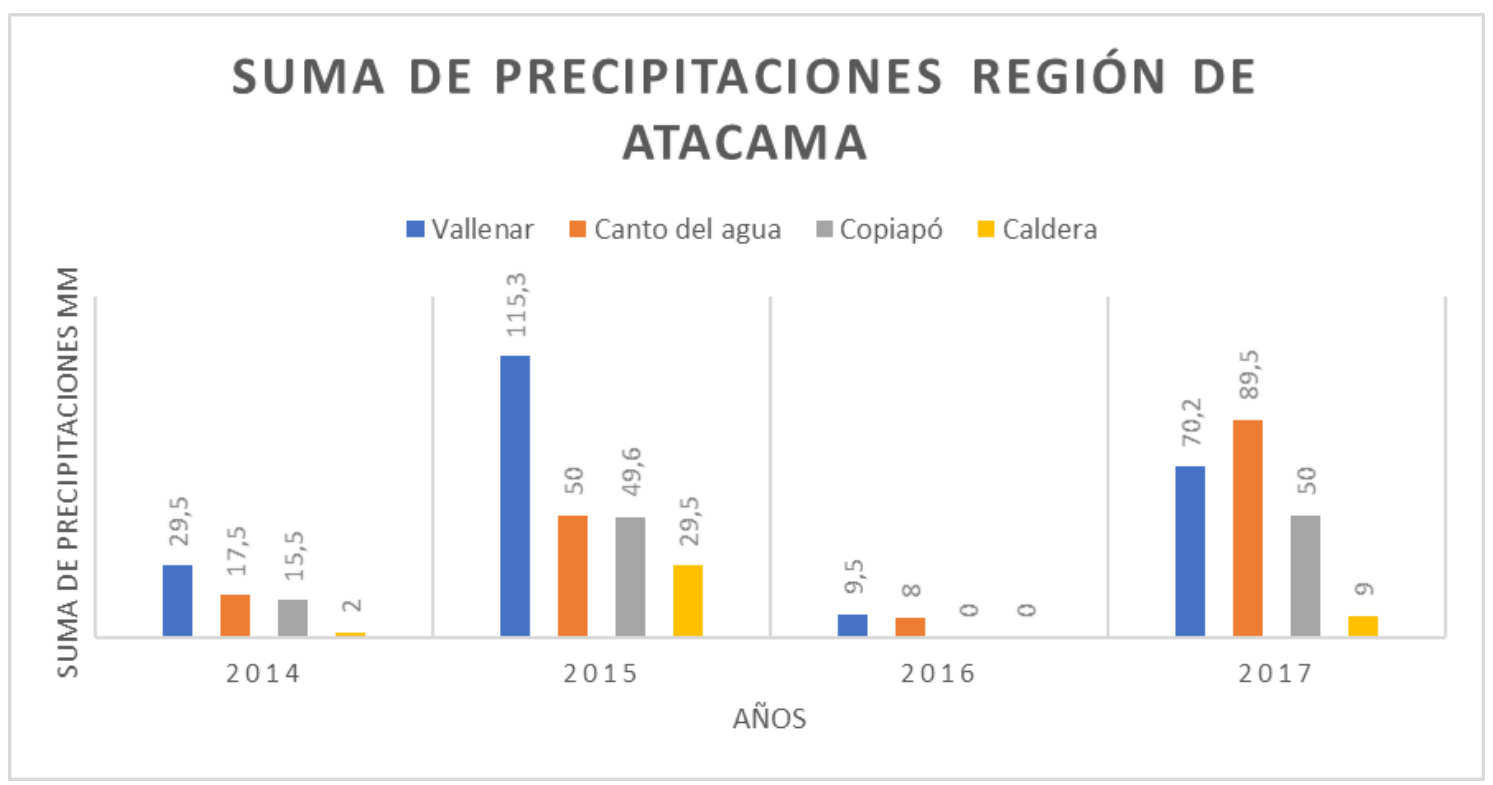

Figura 5. Distribución de la suma precipitación entre los años 2014 y 2017

Fuente: Elaboración propia.

Se realizaron salidas a terrenos para monitoreo in situ del fenómeno del desierto florido. Durante los años 2014 y 2016 no se observó floración. No obstante, durante los años 2015 y 2017 logro evidenciarse floración de distintos tipos (Tabla 1).

Tabla 1. Puntos de observación in situ del fenómeno del desierto florido.

\begin{tabular}{|c|c|c|c|c|c|c|c|}
\hline $\begin{array}{c}\text { Puntos de } \\
\text { terreno }\end{array}$ & 1 & 2 & 3 & 4 & 5 & 6 & 7 \\
\hline Longitud & $70.747049^{\circ}$ & $71.082138^{\circ}$ & 70.531 .639 & 71.147 .510 & 70.926 .845 & 70.706 .533 & 70.608 .489 \\
\hline Latitud & $28.429510^{\circ}$ & $28.554868^{\circ}$ & 27.897.549 & 28.188 .440 & 28.153 .210 & 27.021 .218 & 26.356 .892 \\
\hline $\begin{array}{l}\text { Nombre } \\
\text { Lugar }\end{array}$ & $\begin{array}{l}\text { Camino } \\
\text { Colorado }\end{array}$ & Quebradita & $\begin{array}{r}\text { Vallenar } \\
\text { Ruta } 5\end{array}$ & $\begin{array}{c}\text { Llanos de } \\
\text { Challe }\end{array}$ & $\begin{array}{r}\text { Canto del } \\
\text { Agua }\end{array}$ & $\begin{array}{r}\text { Caldera } \\
\text { Ruta } 5\end{array}$ & Chañaral \\
\hline Visita 2014 & $\begin{array}{r}05 \text { de } \\
\text { agosto }\end{array}$ & $\begin{array}{r}16 \text { de } \\
\text { agosto }\end{array}$ & $\begin{array}{r}05 \mathrm{de} \\
\text { septiembre }\end{array}$ & $\begin{array}{r}06 \text { de } \\
\text { septiembre }\end{array}$ & $\begin{array}{r}08 \mathrm{de} \\
\text { septiembre }\end{array}$ & $\begin{array}{r}15 \text { de } \\
\text { septiembre }\end{array}$ & $\begin{array}{r}21 \mathrm{de} \\
\text { septiembre }\end{array}$ \\
\hline Visita 2015 & $\begin{array}{r}08 \text { de } \\
\text { agosto }\end{array}$ & $\begin{array}{r}19 \text { de } \\
\text { agosto }\end{array}$ & $\begin{array}{r}06 \text { de } \\
\text { septiembre }\end{array}$ & $\begin{array}{r}04 \text { de } \\
\text { septiembre }\end{array}$ & $\begin{array}{r}05 \text { de } \\
\text { septiembre }\end{array}$ & $\begin{array}{r}16 \text { de } \\
\text { septiembre }\end{array}$ & $\begin{array}{r}22 \text { de } \\
\text { septiembre }\end{array}$ \\
\hline Visita 2016 & $\begin{array}{r}09 \text { de } \\
\text { agosto }\end{array}$ & $\begin{array}{r}14 \text { de } \\
\text { agosto }\end{array}$ & $\begin{array}{r}02 \text { de } \\
\text { septiembre }\end{array}$ & $\begin{array}{r}04 \text { de } \\
\text { septiembre }\end{array}$ & $\begin{array}{r}09 \text { de } \\
\text { septiembre }\end{array}$ & $\begin{array}{r}12 \text { de } \\
\text { septiembre }\end{array}$ & $\begin{array}{r}24 \text { de } \\
\text { septiembre }\end{array}$ \\
\hline Visita 2017 & $\begin{array}{r}02 \text { de } \\
\text { agosto }\end{array}$ & $\begin{array}{r}09 \text { de } \\
\text { agosto }\end{array}$ & $\begin{array}{r}01 \text { de } \\
\text { septiembre }\end{array}$ & $\begin{array}{r}02 \text { de } \\
\text { septiembre }\end{array}$ & $\begin{array}{r}07 \mathrm{de} \\
\text { septiembre }\end{array}$ & $\begin{array}{r}14 \text { de } \\
\text { septiembre }\end{array}$ & $\begin{array}{r}12 \mathrm{de} \\
\text { septiembre }\end{array}$ \\
\hline
\end{tabular}

Fuente: Elaboración propias. Los lugares se escogieron debido a la existencia del desierto florido y por su valor NDVI.

Como se indicó en la figura anterior durante el 2014 y 2015 existe una variación considerable de precipitaciones, lo mismo ocurre entre el 2016 y 2017, lo que deriva que al ocurrir estas precipitaciones favorezca mayores valores del índice NDVI, lo que contrastado con información in situ permite atribuir cambios de NDVI en zonas desprovistas normalmente de 


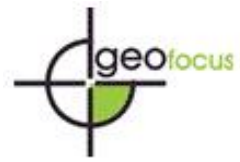

Campos Nazer, J., Espinoza Aburto, J., Ramos Aguilar, J. (2021). Estudio espaciotemporal de la vegetación existente y su relación con el desierto florido en la región de Atacama, Chile. GeoFocus, Revista Internacional de Ciencia y Tecnología de la Información Geográfica (Artículos), 28, 59-93. http://dx.doi.org/10.21138/GF.722

vegetación con una floración extensa, en cambio, años como el 2014 y 2016, la vegetación que existe es solo la productiva asociado a valles fluviales y procesos extractivos, ya que la vegetación presente solo se encuentra distribuida a lo largo y ancho del valle, no así el 2015 y 2017 donde lugares donde no existe ninguna actividad productiva se ven cubiertos de vegetación.

Se identificó un crecimiento en la biota vegetal desde junio, llegando a $188 \mathrm{~km} 2 \mathrm{de}$ vegetación en agosto de 2017, considerando los meses más significativos entre enero y agosto.

Al revisar años donde no existe el fenómeno desierto florido respecto a años donde si existe, es posible evidenciar cambios existentes en área y valores de NDVI presentes en la superficie de la Región de Atacama, donde este cambio y sumado a la información de obtención de datos de terreno, permite atribuir a zonas donde existe el desierto florido (figura 6).
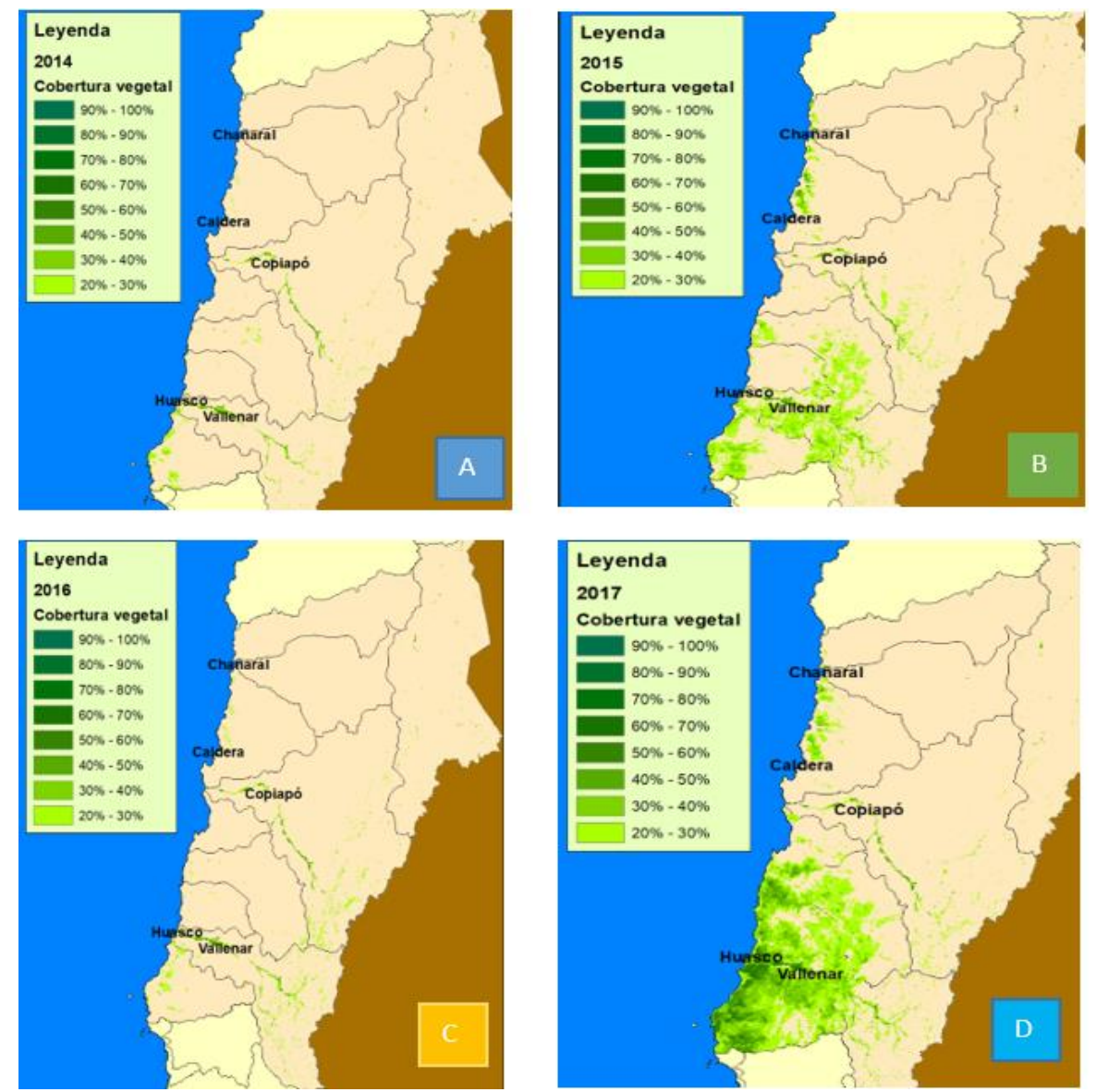

Figura 6. Distribución de la vegetación en distintos años.

A) Cobertura Vegetal año 2014, B) Cobertura Vegetal 2015, C) Cobertura Vegetal 2016, D) Cobertura Vegetal 2017.

Fuente: Elaboración propia.

La cantidad de vegetación representada durante el 2017 es en mayor extensión y mayor factor NDVI que años anteriores, principalmente en la zona Sur de la región, donde se produce un gradiente de precipitación latitudinal, pasando de precipitaciones bajas, o incluso nulas, en el 


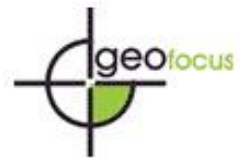

Campos Nazer, J., Espinoza Aburto, J., Ramos Aguilar, J. (2021). Estudio espaciotemporal de la vegetación existente y su relación con el desierto florido en la región de Atacama, Chile. GeoFocus, Revista Internacional de Ciencia y Tecnología de la Información Geográfica (Artículos), 28, 59-93. http://dx.doi.org/10.21138/GF.722

norte de Atacama a mayores precipitaciones en el sur de Atacama, zona de transición desde un clima semiárido y uno mediterráneo ((Luebert \& Pliscoff, 2017; Vanderplank et al., 2014). Esta parte de la región está desprovista de un cauce fluvial que pueda justificar la abundancia, por lo cual gran parte del verdor existente en la región en temporadas específicas, comenzando desde junio en menor medida y aumentando a agosto, es parte del "desierto florido". Este fenómeno fue posible debido a lluvias provocadas por un evento climático extremo, donde un sistema frontal con bajas presiones en superficie y circulación ciclónica en altura adquirió una orientación Suroeste/Noreste debido a un bloqueo existente en la zona sur del país, (Dirección Meteorológica de Chile, 2018).

\section{Conclusiones}

El fenómeno del desierto florido se da con gran intensidad en las zonas costeras, teniendo una gran predominancia en las zonas de la cordillera de la costa de la región de Atacama. Si bien existe una gran extensión de vegetación provocada por las cortas e infrecuentes lluvias, esta biota tiene por lo general una vida corta, ya que las extensiones varían rápidamente de un lugar geográfico a otro.

El año 2017 es el más importante en el promedio de vegetación entre enero hasta agosto de un total de 18 años estudiados. El aumento explosivo de la biota en la región de Atacama se da entre mayo y agosto, luego existe una disminución progresiva. Se demuestra que la mayor concentración de vegetación asociada al desierto florido se da en zonas costeras.

Existe una clara correlación entre precipitación y disponibilidad de biota en la región de atacama sin intervención de un régimen fluvial (Vidiella et al., 1999). Si bien aún no se delimita el desierto florido respecto al resto de biota, se puede atribuir la exposición vegetativa a este particular fenómeno, gracias a la corroboración en terreno es posible evidenciar que zonas desprovistas de vegetación son cubiertas parcialmente por este fenómeno y gracias al factor NDVI permitió seleccionar 7 puntos que fueron registrados durante 4 años consecutivos, evidenciando que años donde aumenta la precipitación, aumenta el factor NDVI, también se evidenció floración.

\section{Referencias bibliográficas}

Almeyda, E. \& Sáez, F. (1958). Recopilación de datos climáticos de Chile. Ministerio de Agricultura, Dirección General de Producción Agraria y Pesquera. Departamento Técnico Interamericano de Cooperación Agrícola.

Armesto, J.; Vidiella, P. \& Gutierrez, J. (1993). Plant communities of the fog-free Chilean coastal desert: plant strategies in a fluctuating environment. Revista Chilena de Historia Natural, n 66, p 271-282.

Castro, C.; Montaña, A.; Pattillo C. \& Zúñiga, A. (2014). Detección del área con desierto florido en el territorio del Mar de Dunas de Atacama, mediante percepción remota. Revista de geografía Norte Grande, no 57, p. 103-121.

Chávez, R.; Moreira-Muñoz, A.; Galleguillos, M.; Olea, M., Aguayo, J.; Latín, A.; AguileraBetti, I.; Muñoz, A.; Manríquez, H. (2019). GIMMS NDVI time series reveal the extent, duration, and intensity of "blooming desert" events in the hyper-arid Atacama Desert, Northern 


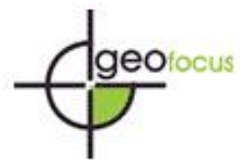

Campos Nazer, J., Espinoza Aburto, J., Ramos Aguilar, J. (2021). Estudio espaciotemporal de la vegetación existente y su relación con el desierto florido en la región de Atacama, Chile. GeoFocus, Revista Internacional de Ciencia y Tecnología de la Información Geográfica (Artículos), 28, 59-93. http://dx.doi.org/10.21138/GF.722

Chile. International Journal of Applied Earth Observation and Geoinformation, vol. 76, p. 193203.

Di Castri, F. \& Hajek, E. R. (1976). Bioclimatología de Chile. Santiago de Chile: Editorial Universidad Católica de Chile.

Dirección de Meteorología de Chile. (2018). Boletín Eventos Extremos. Dirección de servicios Climatológicos. Disponible en internet:

https://climatologia.meteochile.gob.cl/application/publicaciones/boletinEventosExtremos/2017

Engel-Cox, J.; Holloman, C.; Coutant, B. \& Hoff, R. M. (2004) Qualitative and quantitative evaluation of MODIS satellite sensor data for regional and urban scale air quality. Atmospheric environment, vol. 38, no 16, p. 2495-2509.

Fuenzalida, H. (1971). Climatología de Chile. Departamento de Geofísica y Geodesia. Universidad de Chile, 69 pp.

He, B.; Huang, L.; Liu, J.; Wang, H.; Lü, A.; Jiang, W. \& Chen, Z. (2017). The observed cooling effect of desert blooms based on high- resolution Moderate Resolution Imaging Spectroradiometer products. Earth and Space Science, vol. 4, no 5, p. 247-256.

Jaksic, F. (2001). Ecological effects of El Niño in terrestrial ecosystems of western South America, Ecography, vol. 24, no 3, p. 241-250.

Juliá, C.; Montecinos, S.; Maldonado, A. (2008) Características climáticas de la Región de Atacama, en Squeo, F. A. (Ed. 1): Libro rojo de la flora nativa y de los sitios prioritarios para su conservación: Región de Atacama, Copiapó, Ediciones Universidad de La Serena, p. 25-42.

Luebert, F. \& Pliscoff. (2006). Sinopsis bioclimática y vegetacional de Chile. Santiago de Chile: Editorial Universitaria.

Lyapustin, A.; Wang, Y.; Xiong, X.; Meister, G.; Platnick, S.; Levy, R. \& Angal, A. (2014) Scientific impact of MODIS C5 calibration degradation and C6+ improvements, Atmospheric Measurement Techniques, vol. 7, no 12, p. 4353-4365.

Mas, J.F. (2011). Aplicaciones del sensor MODIS para el monitoreo del territorio. México: Editorial Centro de Investigaciones en Geografía Ambiental, Universidad Nacional Autónoma de México.

NASA-MODIS. NASA MODIS Web. Disponible en Internet:

http://modis.gsfc.nasa.gov/about/specifications.php

Noy-Meir, I. (1973). Desert ecosystems: environment and producers. Annual review of ecology and systematics, vol. 4, no 1, p. 25-51.

Rayner, N.; Parker, D.; Horton, E.; Folland, C.; Alexander, L.; Rowell, D.; Kent, E.; Kaplan, A. (2003). Global analyses of sea surface temperature, sea ice, and night marine air temperature since the late nineteenth century, Journal of Geophysical Research: Atmospheres, vol. 108, no D14.

Vanderplank, S.; Moreira-Munoz, A.; Hobohm, C.; Pils, G.; Noroozi, J.; Clark, V.R.; Barker, N.; Yang, W.; Huang, J. \& Ma, K. (2014). Endemism in mainland regions-case studies: Central Chile Ecoregion, Endemism in vascular plants. Springer, Dordrecht, p. 205-308.

Vidiella, P.; Armesto, J.; Gutiérrez, J. (1999). Vegetation changes and sequential flowering after rain in the southern Atacama Desert, Journal of Arid Environments, vol. 43, no 4, p. 449-458. 
